\title{
GENERALIZED OPEN MAPPING THEOREMS FOR BILINEAR MAPS, WITH AN APPLICATION TO OPERATOR ALGEBRAS
}

\author{
P. G. DIXON
}

(Communicated by John B. Conway)

\begin{abstract}
Cohen [4] gave an example of a surjective bilinear mapping between Banach spaces which was not open, and Horowitz [8] gave a much simpler example. We build on Horowitz' example to produce a similar result for bilinear mappings such that every element of the target space is a linear combination of $n$ elements of the range. An immediate application is that Bercovici's construction [1] of an operator algebra with property $\left(\mathbf{A}_{1}\right)$ but not $\left(A_{1}(r)\right)$ can be extended to achieve property $\left(A_{1 / n}\right)$ without $\left(A_{1 / n}(r)\right)$.
\end{abstract}

Let $T: X \times Y \rightarrow Z$ be a continuous bilinear mapping between Banach spaces. We shall be concerned with the following two properties of such a mapping.

DEFINITION. For $n=1,2,3, \ldots$ we say $T$ is $(1 / n)$-surjective if for every $z \in Z$ there exist $x_{1}, x_{2}, \ldots, x_{n} \in X$ and $y_{1}, y_{2}, \ldots, y_{n} \in Y$ with

$$
z=T\left(x_{1}, y_{1}\right)+T\left(x_{2}, y_{2}\right)+\cdots+T\left(x_{n}, y_{n}\right) \text {. }
$$

If, further, there exists a constant $K>0$ such that for every $z$ the $x_{1}, \ldots, x_{n}$ and $y_{1}, \ldots, y_{n}$ may be found, satisfying (1), with

$$
\sum_{i=1}^{n}\left\|x_{i}\right\|\left\|y_{i}\right\| \leq K\|z\|,
$$

then we say that $T$ is $(1 / n)$-open.

REMARKS. (a) These properties are generalizations of the properties $\left(A_{1 / n}\right)$ and " $\left(\mathrm{A}_{1 / n}(r)\right)$ for some $r>0$ " of operator algebras studied by Bercovici, Foias and Pearcy [2]: hence the " $1 / n$ " in our notation.

(b) If $n=1$, "( $1 / n)$-surjective" is "surjective" and " $(1 / n)$-open" is "open".

(c) Notice that

$$
\sum_{i=1}^{n} T\left(x_{i}, y_{i}\right)=\sum_{i=1}^{n} T\left(\lambda_{i} x_{i}, \lambda_{i}^{-1} y_{i}\right)
$$

for any nonzero scalars $\lambda_{1}, \ldots, \lambda_{n}$. We may therefore arrange that $\left\|x_{i}\right\|=\left\|y_{i}\right\|$ $(1 \leq i \leq n)$. Thus, $T$ is $(1 / n)$-open if and only if there is a constant $L>0$ such that every $z \in Z$ with $\|z\| \leq 1$ may be expressed in the form (1) with $\left\|x_{i}\right\| \leq L$, $\left\|y_{i}\right\| \leq L(1 \leq i \leq n)$.

(d) We may work over the real field or the complex field and, in the latter case, we may study either bilinear or sesquilinear maps. The definitions above and Theorems 1 and 2 below apply to all three cases.

Received by the editors September 1, 1987.

1980 Mathematics Subject Classification (1985 Revision). Primary 46A30; Secondary 47D35.

Key words and phrases. Open mapping theorem, bilinear map, dual algebra. 
The best positive result known is the following, which is present, at least implicitly, in [9, Theorem 1.3, 7, Lemma 3.5, 3 and 5].

THEOREM 1. Suppose $T: X \times Y \rightarrow Z$ is a continuous bilinear (or sesquilinear) map between separable Banach spaces $X, Y, Z$. Then

(a) if, for every $z \in Z$, there exists a positive integer $n$ and $x_{1}, \ldots, x_{n} \in X$, $y_{1}, \ldots, y_{n} \in Y$ satisfying (1), then $T$ is $(1 / n)$-surjective for some $n$ (i.e. the dependence of $n$ on $z$ can be removed);

(b) if $T$ is $(1 / n)$-surjective, then $T$ is $(1 / 2 n)$-open.

It is known [6] that the separability condition cannot be removed from (a). We conjecture that it cannot be removed from (b) either. Horowitz [8] showed that, even for finite-dimensional $X, Y, Z$, (b) cannot be improved to "surjective implies open" in the $n=1$ case. Our main result here is the extension of his example to show that, generally, $(1 / n)$-surjective does not imply $(1 / n)$-open. It remains an interesting open question whether $(1 / n)$-surjective implies $(1 / m)$-open for $n<m<2 n$.

THEOREM 2. For each positive integer $n$, there exists $a(1 / n)$-surjective sesquilinear (or bilinear) map $T: \mathbb{C}^{n+2} \times \mathbb{C}^{n+2} \rightarrow \mathbb{C}^{N}$ (where $\left.N=n^{2}+n+2\right)$, which is not $(1 / n)$-open.

PROOF. We show the existence of a sesquilinear map $T$ : a complex bilinear map $\tilde{T}$ with the same properties is then obtained by:

$$
\tilde{T}(x, y):=T(x, \bar{y}) \quad\left(x, y \in \mathbb{C}^{n+2}\right)
$$

and a real bilinear map is obtained just by replacing $\mathbb{C}$ by $\mathbf{R}$.

We observe that a sesquilinear map $T: X \times Y \rightarrow Z$ is $(1 / n)$-surjective (respectively, $(1 / n)$-open), if and only if the map $T^{(n)}: X^{n} \times Y^{n} \rightarrow Z$ is surjective (respectively, open), where

$$
T^{(n)}\left(\left(x_{i}\right)_{i=1}^{n},\left(y_{i}\right)_{i=1}^{n}\right):=\sum_{i=1}^{n} T\left(x_{i}, y_{i}\right) .
$$

Our map $T: \mathbb{C}^{n+2} \times \mathbb{C}^{n+2} \rightarrow \mathbb{C}^{N}$ is defined by

$$
\begin{aligned}
& T\left(\left(u_{1}, \ldots, u_{n}, v, w\right),\left(x_{1}, \ldots, x_{n}, y, z\right)\right) \\
& \quad=\left(\left(u_{i} \overline{x_{j}}\right)_{i, j=1}^{n},\left(u_{i} \bar{y}\right)_{i=1}^{n}, u_{1} \bar{z}+v \overline{x_{1}}+w \bar{y}, w \overline{x_{1}}+v \bar{y}\right) .
\end{aligned}
$$

The reader may verify that in the case $n=1$ this reduces to Horowitz' example, except for the complex conjugates and order of components. To construct $T^{(n)}$ we simply replace the numbers $u_{1}, \ldots, z \in \mathbb{C}$ by vectors $\mathbf{u}_{1}, \ldots, \mathbf{z} \in \mathbb{C}^{n}$ and the products $u_{i} \overline{x_{j}}$, etc., by scalar products $\mathbf{u}_{i} \cdot \mathbf{x}_{j}$, etc.:

$$
\begin{aligned}
& T^{(n)}\left(\left(\mathbf{u}_{1}, \ldots, \mathbf{u}_{n}, \mathbf{v}, \mathbf{w}\right),\left(\mathbf{x}_{1}, \ldots, \mathbf{x}_{n}, \mathbf{y}, \mathbf{z}\right)\right) \\
& \quad=\left(\left(\mathbf{u}_{i} \cdot \mathbf{x}_{j}\right)_{i, j=1}^{n},\left(\mathbf{u}_{i} \cdot \mathbf{y}\right)_{i=1}^{n}, \mathbf{u}_{1} \cdot \mathbf{z}+\mathbf{v} \cdot \mathbf{x}_{1}+\mathbf{w} \cdot \mathbf{y}, \mathbf{w} \cdot \mathbf{x}_{1}+\mathbf{v} \cdot \mathbf{y}\right) .
\end{aligned}
$$

To show that $T^{(n)}$ is surjective, take any vector

$$
\mathbf{h}=\left(\left(\xi_{i j}\right)_{i, j=1}^{n},\left(\eta_{i}\right)_{i=1}^{n}, \varsigma, \theta\right) \in \mathbb{C}^{N}
$$

If $\eta_{i}=0$ for all $i$, then let

$$
\mathbf{x}_{j}=\mathbf{e}_{j} \quad(1 \leq j \leq n)
$$


where $\left(\mathbf{e}_{j}\right)_{i}=1$ if $i=j, 0$ if $i \neq j$

$$
\begin{aligned}
\mathbf{y} & =\mathbf{0} \\
\mathbf{z} & =\mathbf{0} \\
\mathbf{u}_{i} & =\left(\xi_{i 1}, \xi_{i 2}, \ldots, \xi_{\text {in }}\right) \quad(1 \leq i \leq n) \\
\mathbf{v} & =(\varsigma, 0,0, \ldots, 0) \\
\mathbf{w} & =(\theta, 0,0, \ldots, 0)
\end{aligned}
$$

otherwise, let

$$
\begin{array}{rlrl}
\mathbf{u}_{i} & =\mathbf{e}_{i} & & (1 \leq i \leq n) \\
\mathbf{x}_{j} & =\left(\bar{\xi}_{1 j}, \bar{\xi}_{2 j}, \ldots, \bar{\xi}_{n j}\right) & & (1 \leq j \leq n), \\
\mathbf{y} & =\left(\bar{\eta}_{1}, \bar{\eta}_{2}, \ldots, \bar{\eta}_{n}\right), & \\
\mathbf{v} & =\theta(\mathbf{y} \cdot \mathbf{y})^{-1} \mathbf{y}, & \\
\mathbf{w} & =\mathbf{0} \\
\mathbf{z} & =\left(\varsigma-\left(\mathbf{v} \cdot \mathbf{x}_{1}\right)\right)^{-} \mathbf{u}_{1} . &
\end{array}
$$

In both cases, $T^{(n)}\left(\left(\mathbf{u}_{1}, \ldots, \mathbf{w}\right),\left(\mathbf{x}_{1}, \ldots, \mathbf{z}\right)\right)=\mathbf{h}$. This proves that $T^{(n)}$ is surjective, so $T$ is $(1 / n)$-surjective.

To show that $T$ is not $(1 / n)$-open, suppose otherwise. Then, by Remark (c) and the equivalence of norms on finite-dimensional spaces, there exists a number $M \geq 1$ such that, whenever $\mathbf{h}=\left(\left(\xi_{i j}\right),\left(\eta_{i}\right), \varsigma, \theta\right) \in \mathbb{C}^{N}$ with $\left|\xi_{i j}\right|,\left|\eta_{i}\right|,|\varsigma|,|\theta| \leq 1$ for all $i$, $j$, there exist $\mathbf{u}_{i}, \mathbf{v}, \mathbf{w}, \mathbf{x}_{i}, \mathbf{y}, \mathbf{z} \in \mathbb{C}^{n}(1 \leq i \leq n)$ such that

$$
T^{(n)}\left(\left(\mathbf{u}_{1}, \ldots, \mathbf{u}_{n}, \mathbf{v}, \mathbf{w}\right),\left(\mathbf{x}_{1}, \ldots, \mathbf{x}_{n}, \mathbf{y}, \mathbf{z}\right)\right)=\mathbf{h}
$$

and

$$
\left\|\mathbf{u}_{i}\right\|,\|\mathbf{v}\|,\|\mathbf{w}\|,\left\|\mathbf{x}_{i}\right\|,\|\mathbf{y}\|,\|\mathbf{z}\| \leq M \quad(1 \leq i \leq n),
$$

where $\|\cdot\|$ denotes the Euclidean norm: $\|x\|:=(x \cdot x)^{1 / 2}\left(x \in \mathbb{C}^{n}\right)$.

Extending Horowitz' argument, we let $\mathbf{h}$ be defined by:

$$
\begin{aligned}
& \xi_{11}=a, \quad \xi_{i i}=1 \quad(2 \leq i \leq n), \quad \xi_{i j}=0 \quad(i \neq j), \\
& \eta_{1}=a, \quad \eta_{i}=0 \quad(2 \leq i \leq n), \\
& \varsigma=a, \quad \theta=1
\end{aligned}
$$

where $0<a<1$ and we shall eventually make $a$ tend to zero. Suppose $\mathbf{u}_{i}, \mathbf{v}, \mathbf{w}$, $\mathbf{x}_{i}, \mathbf{y}, \mathbf{z}$ satisfy (2) and (3) above. We wish to show that $\left\|\mathbf{x}_{1}\right\|\left\|\mathbf{u}_{1}\right\|$ must be small. This is most conveniently done by the following matrix argument.

Define the $n \times n$ matrices $\mathbf{A}=\left(\mathbf{a}_{1}, \mathbf{a}_{2}, \ldots, \mathbf{a}_{n}\right), \mathbf{B}=\left(\mathbf{b}_{1}, \mathbf{b}_{2}, \ldots, \mathbf{b}_{n}\right)$ from the column vectors

$$
\begin{array}{lll}
\mathbf{a}_{1}=a^{-1 / 2} \mathbf{u}_{1}, & \mathbf{a}_{i}=\mathbf{u}_{i} & (2 \leq i \leq n) \\
\mathbf{b}_{1}=a^{-1 / 2} \mathbf{x}_{1}, & \mathbf{b}_{j}=\mathbf{x}_{j} & (2 \leq j \leq n)
\end{array}
$$


Then the equations $\mathbf{u}_{i} \cdot \mathbf{x}_{j}=\xi_{i j}(1 \leq i, j \leq n)$ become $\mathbf{A}^{*} \mathbf{B}=\mathbf{I}$. It follows that $\mathbf{A}$ and $\mathbf{B}$ are invertible and $\mathbf{B} \mathbf{A}^{*}=\mathbf{I}=\mathbf{B}^{*} \mathbf{A}$, so $\mathbf{B}^{*} \mathbf{B} \mathbf{A}^{*} \mathbf{A}=\mathbf{I}$. Hence

$$
\begin{aligned}
1 & =\left(\mathbf{B}^{*} \mathbf{B} \mathbf{A}^{*} \mathbf{A}\right)_{11} \\
& =\sum_{i=1}^{n}\left(\mathbf{b}_{i} \cdot \mathbf{b}_{1}\right)\left(\mathbf{a}_{1} \cdot \mathbf{a}_{i}\right) \\
& =a^{-2}\left\|\mathbf{x}_{1}\right\|^{2}\left\|\mathbf{u}_{1}\right\|^{2}+\sum_{i=2}^{n} a^{-1}\left(\mathbf{x}_{i} \cdot \mathbf{x}_{1}\right)\left(\mathbf{u}_{1} \cdot \mathbf{u}_{i}\right) .
\end{aligned}
$$

Since $\left\|\mathbf{x}_{i}\right\|,\left\|\mathbf{u}_{i}\right\| \leq M(1 \leq i \leq n)$, this yields

$$
a^{-2}\left\|\mathbf{x}_{1}\right\|^{2}\left\|\mathbf{u}_{1}\right\|^{2} \leq 1+(n-1) a^{-1}\left\|\mathbf{x}_{1}\right\|\left\|\mathbf{u}_{1}\right\| M^{2} \text {. }
$$

Hence

$$
\left\|\mathbf{x}_{1}\right\|\left\|\mathbf{u}_{1}\right\| \leq a\left(1+(n-1) M^{2}\right) \leq a n M^{2} .
$$

Next, since $A$ is invertible, $\left(\mathbf{u}_{1}, \mathbf{u}_{2}, \ldots, \mathbf{u}_{n}\right)$ is a basis for $\mathbb{C}^{n}$. Now $\mathbf{u}_{i} \cdot\left(\mathbf{x}_{1}-\mathbf{y}\right)=0$ for all $i$, so it follows that $\mathbf{x}_{1}=\mathbf{y}$. Then the equation $\mathbf{w} \cdot \mathbf{x}_{1}+\mathbf{v} \cdot \mathbf{y}=\theta=1$ gives

$$
(\mathbf{w}+\mathbf{v}) \cdot \mathbf{x}_{1}=1 \text {, }
$$

and so

$$
\|\mathbf{w}+\mathbf{v}\|\left\|\mathbf{x}_{1}\right\| \geq 1
$$

We also have

$$
\mathbf{u}_{1} \cdot \mathbf{z}+\mathbf{v} \cdot \mathbf{x}_{1}+\mathbf{w} \cdot \mathbf{y}=\varsigma=a
$$

so

$$
\mathbf{u}_{1} \cdot \mathbf{z}=a-(\mathbf{v}+\mathbf{w}) \cdot \mathbf{x}_{1}=-(1-a)
$$

by (5), and so

$$
\left\|\mathbf{u}_{1}\right\| \geq(1-a) / M
$$

Combining (4) and (7),

$$
\left\|\mathbf{x}_{1}\right\| \leq a n M^{3} /(1-a) .
$$

Finally,

$$
\begin{aligned}
2 M & \geq\|\mathbf{w}+\mathbf{v}\| \\
& \geq\left\|\mathbf{x}_{1}\right\|^{-1}, \quad \text { by }(6), \\
& \geq(1-a) / a n M^{3}, \quad \text { by }(8) .
\end{aligned}
$$

Now $M$ is a constant, independent of $a$, so letting $a$ tend to zero gives the required contradiction, completing the proof of Theorem 2.

COROLlARY. For every positive integer $n$, there is a commutative $\left(n^{2}+n+3\right)$ dimensional algebra of operators on the Hilbert space $\mathbb{C}^{2 n+5}$ which has property $\left(A_{1 / n}\right)$, but which, for all $r>0$, does not have property $\left(A_{1 / n}(r)\right)$.

The proof of this is the same as that of [1], where Bercovici derives this result for $n=1$ from Horowitz' example. We therefore omit further discussion and refer the reader to [1] for an explanation of the terminology as well as for the proof. 


\section{REFERENCES}

1. H. Bercovici, Note on property $\left(A_{1}\right)$, Linear Algebra Appl. 91 (1987), 213-216.

2. H. Bercovici, C. Foias and C. Pearcy, Dual algebras with applications to invariant subspaces and dilation theory, CBMS Regional Conf. Ser. in Math., no. 56, Amer. Math. Soc., Providence, R. I., 1985.

3. B. Chevreau and J. Esterle, Pettis' lemma and topological properties of dual algebras, Michigan Math. J. 34 (1987), 143-146.

4. P. J. Cohen, A counterexample to the closed graph theorem for bilinear maps, J. Funct. Anal. 16 (1974), 235-239.

5. H. G. Dales, Automatic continuity theory (in preparation).

6. P. G. Dixon, Non-separable Banach algebras whose squares are pathological, J. Funct. Anal. 26 (1977), 190-200.

7. _ Automatic continuity of positive functionals on topological involution algebras, Bull. Austral. Math. Soc. 23 (1981), 265-281.

8. C. Horowitz, An elementary counterexample to the open mapping principle for bilinear maps, Proc. Amer. Math. Soc. 53 (1975), 293-294.

9. R. J. Loy, Multilinear mappings and Banach algebras, J. London Math. Soc. (2) 14 (1976), 423-429.

Department of Pure Mathematics, University of Sheffield, Sheffield, S3 7RH, ENGLAND 